\title{
Mathematik inklusiv unterrichten - Ein Forschungsüberblick zum aktuellen Stand der Entwicklung einer inklusiven Didaktik für den Mathematikunterricht in der Grundschule
}

\author{
Hannah Jütte $\cdot$ Miriam M. Lüken (D)
}

Eingegangen: 5. Juni 2020 / Angenommen: 11. Oktober 2020 / Online publiziert: 14. Januar 2021

(C) Der/die Autor(en) 2021

Zusammenfassung Der Mathematikunterricht der Grundschule hat sich in den letzten Jahren im Hinblick auf die Realisierung einer inklusiven Didaktik weiterentwickelt. Es herrscht Konsens, dass alle Kinder mit- und voneinander lernen, jedoch auch individuell gefördert werden sollen. Forschungsergebnisse zeigen neutrale bis positive Effekte inklusiven Unterrichts auf das kindliche Mathematiklernen. Aktuelle Studien weisen jedoch darauf hin, dass für Lehrkräfte die Realisierung gemeinsamer Lernsituationen aufgrund fachlicher Besonderheiten nach wie vor herausfordernd ist. Trotz einiger konkretisierter mathematikdidaktischer Konzepte, die im Beitrag vorgestellt werden, besteht daher weiterer Forschungs- und Entwicklungsbedarf zur Gestaltung inklusiven Mathematikunterrichts auf inhaltlicher Ebene.

Schlüsselwörter Inklusiver Mathematikunterricht · Grundschulmathematik · Unterrichtsforschung · Mathematikdidaktische Konzepte · Inklusive Mathematikdidaktik

H. Jütte $(\bowtie) \cdot$ Prof. Dr. M. M. Lüken Institut für Didaktik der Mathematik, Universität Bielefeld, Universitätsstraße 25, 33615 Bielefeld, Deutschland

E-Mail: hannah.juette@uni-bielefeld.de

Prof. Dr. M. M. Lüken

E-Mail: miriam.lueken@uni-bielefeld.de 


\title{
Teaching math inclusive-A research review on recent developments of an inclusive didactic for mathematics lessons in primary school
}

\begin{abstract}
In recent years, an inclusive didactic for mathematics lessons in primary school has been further developed. It is widely recognized that all children should learn with each other and from another, and additionally should be supported individually. Research shows neutral to positive effects of inclusive education on children's mathematical development. However, recent studies indicate that for teachers, realizing joint learning is challenging because of the contents nature. Despite of few substantiated didactical concepts for mathematics education, which are reviewed in this article, we call for further research and development in the organization of inclusive mathematics learning on a content-related level.
\end{abstract}

Keywords Inclusive mathematics lesson · Primary mathematics · Classroom research · Concepts for mathematics education - Inclusive mathematics education

\section{Einleitung}

„Mathematik ist ein schlechtes Fach für den gemeinsamen Unterricht“ (zit. nach Korff 2015, S. 205). Diese in ähnlicher Form immer wieder zu hörende Aussage einer Lehrkraft aus der Interviewstudie von Korff (2015) verdeutlicht, dass die Gestaltung eines inklusiven Mathematikunterrichts scheinbar mit besonderen Herausforderungen verbunden ist.

Mathematisches Wissen ist mehr als nur rechnen zu können. Im aktuellen Mathematikunterricht der Grundschule sollen Kinder neben arithmetischen Kompetenzen auch Kompetenzen im Bereich der Geometrie erwerben, mit mathematischen Größen wie Längen, Gewichten und Volumina messen und rechnen, ein erstes Verständnis für Wahrscheinlichkeiten entwickeln und in der Auseinandersetzung mit diesen Inhalten die der Mathematik zugrundeliegenden Muster und Strukturen nutzen (KMK 2004, S. 8). Dabei steht nicht nur das Anwenden können von Prozeduren, sondern vor allem die Entwicklung eines gesicherten Verständnisses mathematischer Inhalte im Fokus des aktuellen Mathematikunterrichts. Einen weiteren Schwerpunkt stellen daher mathematische Tätigkeiten dar, die als allgemeine Kompetenzen bezeichnet werden: Kinder lösen inner- und außermathematische Probleme, sie tauschen mathematische Argumente aus, sprechen über Mathematik und stellen sie dar (ebd., S. $6 \mathrm{ff}$.). Entscheidend ist, dass mathematische Inhalte nicht nur individuell von den Lernenden konstruiert werden, sondern dass die individuellen Konstruktionen in Wechselwirkung mit Kooperation und Kommunikation stehen. Eine inhaltliche Auseinandersetzung mit anderen Kindern trägt wesentlich zum Erlernen von neuem mathematischen Wissen bei (Fetzer 2019, S. 23). Fachliche Besonderheiten liegen im hierarchischen Aufbau der Inhalte sowie in der Abstraktheit mathematischer Objekte. Das bedeutet zum einen, dass grundlegende Kompetenzen erforderlich sind, um auf einem höheren Niveau weiterarbeiten zu können (ebd., S. 5) und zum anderen, dass die Auseinandersetzung mit Mathematik auf abstrakte Objekte und Konzepte abzielt. Diese können nicht einfach gezeigt werden, sondern bedürfen der 
Vermittlung durch Veranschaulichungen, müssen also aus konkreten Darstellungen und Handlungen entwickelt werden (Bruner 1974; vom Hofe 1995).

Ist ein inklusiver Mathematikunterricht - verstanden nicht allein als ein räumliches Beisammensein von Lernenden mit und ohne einen sonderpädagogischen Unterstützungsbedarf (kurz: SPU) in einem gemeinsamen Klassenzimmer, sondern als ein Arbeiten und Austauschen im Sinne von Feuser (1998) an einem gemeinsamen Gegenstand - unter diesen fachlichen Voraussetzungen überhaupt möglich und effektiv für alle Kinder? ${ }^{1}$ Welche (evaluierten) Konzepte wurden in der Mathematikdidaktik bisher für einen inklusiven Unterricht entwickelt? Und welche besonderen Herausforderungen ergeben sich gegenwärtig bei der Gestaltung des Unterrichts? Diesen Fragen geht der vorliegende Beitrag im Diskurs um die Etablierung eines inklusiven Unterrichts im Fach Mathematik der Grundschule nach. Zu Beginn geben wir einen Überblick zu den Effekten inklusiven Unterrichts auf die mathematischen Kompetenzen von Kindern mit und ohne SPU². Anschließend werden anschlussfähige Herangehensweisen und Überlegungen in Form verschiedener mathematikdidaktischer Konzepte für die Gestaltung eines inklusiven Unterrichts im Spannungsfeld zwischen individueller Förderung und gemeinsamem Lernen aufgezeigt sowie empirische Befunde zur aktuellen Praxis inklusiven Mathematikunterrichts dargelegt.

\section{Effekte inklusiven Unterrichts auf das kindliche Mathematiklernen}

In den letzten 60 Jahren konnten zahlreiche Studien neutrale bis positive Wirkungen eines inklusiven Unterrichts auf die Schulleistungen von Kindern mit SPU zeigen. Bei genauerem Hinsehen wird jedoch deutlich, dass Mathematikleistungen nicht in allen Studien erhoben wurden, sondern sich die Schulleistungen auf Lese- oder Rechtschreibfähigkeiten bzw. allgemeine kognitive Fähigkeiten beziehen. Außerdem ergaben sich je nach Art des Unterstützungsbedarfs insbesondere für das Fach Mathematik teilweise andere Effekte als im sprachlichen Bereich. Im Folgenden geben wir daher einen fachlich fokussierten und soweit möglich nach Unterstützungsbedarfen differenzierten Überblick zu Effekten inklusiven Unterrichts auf das kindliche Mathematiklernen in der Grundschule. Wir ziehen dabei nur Studien mit parallelisierten Vergleichsgruppen heran, in denen jeweils ein Kind aus inklusiver Beschulung in zahlreichen Merkmalen wie Geschlecht, sozioökonomischem Status und Intelligenz einem Kind mit identischen Merkmalsausprägungen zugeordnet wird, das nicht inklusiv unterrichtet wird. Mit inklusivem Unterricht ist in diesem Kapitel als Minimalanforderung das räumliche Miteinander von Kindern mit und

\footnotetext{
1 In Deutschland wurde Integration, im Gegensatz zu vielen anderen westlichen Ländern, lange Zeit vorrangig als räumliches Beisammensein von Kindern mit und ohne SPU verstanden. Erst mit der UN-Behindertenrechtskonvention im Jahr 2009 entwickelte sich der Inklusionsbegriff und Formen des gemeinsamen Lernens wurden zunehmend im Unterricht etabliert.

${ }^{2}$ Im gesamten Beitrag werden für die einzelnen sonderpädagogischen Unterstützungsbedarfe folgende Abkürzungen verwendet: Lernen (LE), Emotionale und soziale Entwicklung (ESE), Geistige Entwicklung (GG), Sprache (SQ), Sehen (SE). Die Abkürzungen orientieren sich an den Vorgaben des Ministeriums für Schule und Bildung des Landes NRW (MSB NRW o.J.).
} 
ohne SPU im Unterricht gemeint. Der Umfang des tatsächlichen gemeinsamen Lernens wird in den meisten Studien nicht expliziert.

\subsection{Effekte inklusiven Unterrichts auf die mathematische Entwicklung von Kindern mit SPU}

Die Ergebnisse vieler nationaler sowie internationaler Studien zeigen, dass sich die mathematischen Kompetenzen von Kindern mit dem SPU LE und dem SPU ESE, aber auch von Kindern mit leichten geistigen Behinderungen, in einem inklusiven Unterricht stärker entwickeln als in einem separativen Setting (Karsten et al. 2001, NL; Peetsma et al. 2001, NL; Saint-Laurent et al. 1998, CA; Stevens und Slavin 1995, US). Der in der Querschnittstudie von Kocaj et al. (2014, D) identifizierte Unterschied in den mathematischen Kompetenzen von Kindern mit dem SPU LE an Grundschulen entsprach dabei Ende Klasse 4 einem Leistungsvorsprung von fast einem Schuljahr. In den Längsschnittstudien von Haeberlin et al. (1999, CH) und Bless (1995, CH) machten Kinder mit dem SPU LE in inklusiven Klassen Lernfortschritte, die bis zu doppelt so groß waren wie die Lernfortschritte von Kindern in Förderschulklassen. Vergleicht man unterschiedliche Fächer, zeigt sich darüber hinaus, dass die positiven Effekte inklusiven Unterrichts in den Mathematikleistungen größer sind als im sprachlichen Bereich (Affleck et al. 1988, US; Bear und Proctor 1990, US; Bless 1995, CH; Haeberlin et al. 1999, CH; Kocaj et al. 2014, D).

Im Gegensatz zu den obigen Studien fanden Cole et al. (2004, US) sowie Waldron und McLeskey (1998, US) nur vergleichbare Lernfortschritte von Kindern mit dem SPU LE in einem inklusiven versus einem separativen Unterricht. Den gleichen Befund zeigen die Studien zu inklusiver Beschulung von Kindern mit dem SPU GG und SPU SQ: Im Bereich Mathematik konnten keine Unterschiede in der Leistungsentwicklung zwischen inklusiven Klassen und Sonderschulklassen gezeigt werden, obwohl teilweise positive Effekte zugunsten der inklusiv unterrichteten Kinder im sprachlichen Bereich festgestellt wurden (Budoff und Gottlieb 1976, US; Cole et al. 2004, US; Kocaj et al. 2014, D; Sermier Dessemontet et al. 2012, CH; siehe auch Schnepel 2019).

\subsection{Effekte inklusiven Unterrichts auf die mathematische Entwicklung von Kindern ohne SPU}

Eine beträchtliche Anzahl an Studien untersuchte den Einfluss inklusiven Unterrichts an Grundschulen auf die Schulleistungen der Mitschüler*innen, also Kinder ohne SPU (für einen Überblick siehe Ruijs und Peetsma 2009). Demnach scheint ein inklusiver Unterricht mit Kindern mit dem SPU GG keine Auswirkungen auf die Mathematikleistungen ihrer Mitschüler*innen zu haben (Hunt et al. 1994, US; McDonnell et al. 2003, US). Für einen gemeinsamen Unterricht mit Kindern mit dem SPU LE zeigten entsprechende Studien neutrale (Affleck et al. 1988, US; Willrodt und Claybrook 1995, US) bis positive Effekte auf die Mathematikleistungen der Grundschulkinder ohne SPU (Cole et al. 2004, US; Saint-Laurent et al. 1998, CA; Stevens und Slavin 1995, US; Wocken 1987, D). Huber et al. (2001, US) zeigten differenziert, dass Kinder mit geringen mathematischen Kompetenzen von einem 
gemeinsamen Mathematikunterricht mit Kindern mit dem SPU LE profitieren, wohingegen Kinder mit überdurchschnittlichen mathematischen Kompetenzen in diesem Setting kleinere Fortschritte in ihrer Lernentwicklung machen. Im Gegensatz dazu fanden Stevens und Slavin (1995, US) auch für die Kinder mit überdurchschnittlichen mathematischen Kompetenzen eine positivere Lernentwicklung, wenn der inklusive Mathematikunterricht auf ein mit- und voneinander Lernen ausgerichtet ist. Für Auswirkungen eines inklusiven Unterrichts mit Kindern mit anderen Unterstützungsbedarfen gibt es keine Einzelstudien, sie sind jedoch als kleine Teilstichproben in vielen Studien enthalten (Affleck et al. 1988, US; Hunt et al. 1994, US; McDonnell et al. 2003, US; Willrodt und Claybrook 1995, US; Saint-Laurent et al. 1998, CA) und die Ergebnisse ähneln denen der Kinder mit dem SPU LE.

\subsection{Zusammenfassung}

Die referierten Studien unterscheiden sich stark in der organisatorischen, personellen sowie inhaltlichen Ausgestaltung des inklusiven Unterrichts und sind in verschiedene Forschungssettings eingebettet. Dies könnte die zum Teil unterschiedlichen Ergebnisse erklären. Dennoch kann aufgrund der Studienlage angenommen werden, dass sich sowohl Kinder mit als auch ohne SPU in einem gemeinsamen Mathematikunterricht mindestens genauso gut mathematisch entwickeln, wie in traditionellen, separativen Settings. Ein positiver Effekt der Inklusion auf die Lernentwicklung scheint umso größer zu sein, je häufiger gemeinsames Lernen stattfindet (Freeman und Alkin 2000). Außerdem scheint die Dauer der gemeinsamen Beschulung bedeutsam zu sein. Effekte zeigten sich teilweise erst nach vier Jahren gemeinsamen Unterrichts (Budoff und Gottlieb 1976; Peetsma et al. 2001).

Aus mathematikdidaktischer Perspektive ist kritisch anzumerken, dass Kocaj et al. (2014) die Einzigen sind, die für die Mathematikleistung einen Globalwert mit Kompetenzen aller fünf inhaltsbezogenen Kompetenzbereiche der Bildungsstandards Mathematik für die Grundschule bildeten. Die übrigen referierten Studien testeten ausschließlich arithmetische Fähigkeiten, was dem aktuellen Bild von relevanten mathematischen Kompetenzen widerspricht (KMK 2004). Folglich besteht Forschungsbedarf dahingehend, auch geometrische Lernentwicklungen oder die Entwicklung des Größenverständnisses von Kindern mit SPU im inklusiven Unterricht zu verfolgen. Darüber hinaus ist noch unklar, inwieweit Kinder mit SPU beim gemeinsamen Lernen allgemeinen Kompetenzen erwerben. Wichtiger Forschungsbedarf besteht außerdem im Hinblick auf die Frage, wie ein inklusiver Mathematikunterricht für Kinder mit und ohne SPU im Spannungsfeld zwischen gemeinsamem, ko-konstruktiven Lernen und individueller Förderung im Detail ausgestaltet werden sollte, um einen Lernzuwachs für alle Kinder sichern zu können (Kocaj et al. 2014). Darüber geben die betrachteten Studien nur sehr eingeschränkt Auskunft. 


\section{3 Übergeordnete Konzeptionen und ausgearbeitete Konzepte zur Gestaltung eines inklusiven Mathematikunterrichts}

Im Folgenden wollen wir die Frage nach der Gestaltung des Unterrichts in den Blick nehmen. Bisher gibt es nur wenige Studien, die konkrete Konzepte ${ }^{3}$ für einen inklusiven Mathematikunterricht entwickelt, erprobt und evaluiert haben. In den letzten Jahren gab es jedoch eine vermehrte Publikationstätigkeit in diesem Bereich. Unterrichtsorganisatorische Überlegungen finden sich beispielsweise bei RathgebSchnierer und Feindt (2014) sowie bei Schindler (2017). Sie zeigen auf konzeptioneller Ebene, wie individuelles und gemeinsames Lernen miteinander vernetzt werden kann. Hinweise und Anregungen, die sich auf die Vermittlung ausgewählter mathematischer Inhalte im Rahmen eines inklusiven Unterrichts beziehen, finden sich unter anderem bei Fetzer (2019), Häsel-Weide und Nührenbörger (2015) sowie Käpnick (2016). Während in diesen didaktischen Konzeptionen das gemeinsame Lernen aller Kinder einer Klasse in den Blick genommen wird, differenzieren andere Konzeptionen die Anforderungen für die unterschiedlichen Unterstützungsbedarfe weiter aus. Für den SPU GG seien hier Moser Opitz (2016), Ratz (2017) und Scherer (2017) genannt. Eine Konzeption zur Auswahl und Adaption von Lernmaterialien für den SPU SE publizierte Leuders (2016) sowie für den SPU SQ Werner und Berg (2014).

\subsection{Gemeinsamkeiten mathematikdidaktischer Konzeptionen: Lernen am gemeinsamen Gegenstand - natürliche Differenzierung - fundamentale Ideen}

Die mathematikdidaktischen Überlegungen im aktuellen Diskurs um die Gestaltung eines inklusiven Unterrichts haben gemein, dass sie (1) das Lernen am gemeinsamen Gegenstand nach Feuser (1998) aufgreifen, dabei (2) innere Differenzierungsmaßnahmen mitdenken und (3) sich - um das Spannungsfeld zwischen gemeinsam lernen und effektiv fördern bewältigen zu können (Moser Opitz 2014, S. 65) - fachlich an fundamentalen Ideen der Mathematik orientieren. Der erste Punkt, das gemeinsame Lernen, ist für den Mathematikunterricht nicht neu, sondern gehört durch die kokonstruktive Sichtweise von Mathematiklernen zum Grundverständnis eines zeitgemäßen Mathematikunterrichts. Ebenso sind die allgemeinen Kompetenzen der Bildungsstandards für das Fach Mathematik ein Abbild dessen, dass „,mathematisches Lernen ein aktiver Prozess [ist], der in sozialen Bezügen stattfindet und sein Potenzial im Miteinander entfaltet“ (Fetzer 2019, S. 9). Grundlage für einen Austausch der Lernenden ist der gemeinsame Gegenstand. Um den individuellen Voraussetzungen und Unterstützungsbedarfen der Kinder gerecht zu werden, sind zusätzlich Differenzierungsmaßnahmen erforderlich.

\footnotetext{
3 Mathematikdidaktische Überlegungen zur Umsetzung einer inklusiven Didaktik werden in der Literatur mit verschiedenen Begriffen verbunden. Der Begriff Konzeption meint im Folgenden übergeordnete, eher organisatorische oder normative Überlegungen und der Begriff Konzept ausgearbeitete Vorschläge für das Unterrichten mathematischer Inhalte.
} 
In der Mathematikdidaktik hat sich als besondere Form der inneren Differenzierung die sogenannte natürliche Differenzierung etabliert. Die natürliche Differenzierung geht auf Wittmann (1990, S. 159) zurück und kann als zentrale mathematikdidaktische Konzeption zum Umgang mit Heterogenität betrachtet werden. Entgegen anderer Formen der Differenzierung, bei denen ein Großteil der Entscheidungen von der Lehrkraft ausgeht und die Lernenden kaum in den inhaltlichen Entscheidungsprozess mit einbezogen werden, kann die natürliche Differenzierung als eine Differenzierung ,,vom Kind aus“ (Wittmann und Müller 2004, S. 15) verstanden werden. Anstelle festgelegter Aufgaben für unterschiedliche Leistungsniveaus gibt es ein inhaltlich ganzheitliches und hinreichend komplexes, für alle Kinder gleiches Lernangebot, bei der sich Aufgaben unterschiedlicher Schwierigkeitsstufen in natürlicher Weise ergeben. Die Lernenden dürfen selbst über Ansatzpunkte, Bearbeitungswege und die Tiefe der Bearbeitung entscheiden. Da alle Kinder an einem gleichen Themenkomplex arbeiten, kann eine inhaltlich gehaltvolle Diskussion bezüglich ihrer Bearbeitungen und Erkenntnisse, im Sinne des mit- und voneinander Lernens, stattfinden (Fetzer 2019, S. 12 ff.; Krauthausen und Scherer 2014, S. 50f.).

Einen Ansatzpunkt für die Identifizierung gemeinsamer Lernsituationen für die hierarchisch angeordneten Inhalte bieten die sogenannten fundamentalen Ideen der Mathematik (Häsel-Weide 2017; Winter 2001; Wittmann 1998), auf die sich viele Konzeptionen beziehen. Eine Orientierung an fundamentalen Ideen meint, dass eine mathematische Idee im Laufe der Schuljahre spiralcurricular bearbeitet wird, denn für einen inklusiven Mathematikunterricht gilt grundsätzlich: Es müssen nicht andere Inhalte behandelt werden, sondern die zentralen Inhalte auf unterschiedlichen Niveaus (Häsel-Weide und Nührenbörger 2017c, S. 12f.). Mit den fundamentalen Ideen werden folglich mathematische Inhalte aufgezeigt, die für die Entwicklung aller Kinder entscheidend sind. Die Inhalte bauen aufeinander auf, werden immer wieder aufgegriffen und können von unterschiedlichen Niveaus aus von jedem Kind erarbeitet werden. Es besteht zwar Konsens über die Bedeutung und das Ziel von fundamentalen Ideen, die Erarbeitung eines mehrheitlich akzeptierten Katalogs dieser Ideen steht jedoch noch aus (Werner 2019, S. 24).

\subsection{Mathematikdidaktische Konzepte für inklusiven Matheunterricht: drei Beispiele}

Mathematikdidaktische Konzeptionen für einen inklusiven Unterricht sind bedeutsam, sie können jedoch nur ein erster Schritt sein. Es sind darüber hinaus evaluierte didaktische Konzepte erforderlich, die Lehrkräften konkret aufzeigen, wie individuelle Förderung, Differenzierung und gemeinsames Lernen an mathematischen Inhalten in heterogenen Lerngruppen gelingen kann. Im Folgenden werden drei Konzepte zur Gestaltung eines inklusiven Mathematikunterrichts vorgestellt. Die Konzepte wurden aufgrund der von Moser Opitz (2014) aus der Theorie als auch der LehrLern-Forschung zum Unterricht in heterogenen Lerngruppen abgeleiteten Kriterien für eine inklusive Didaktik ausgewählt: die optimale Förderung aller Lernenden einer heterogenen Lerngruppe, die Ausweisung von Differenzierungsmöglichkeiten, die Berücksichtigung der Tiefenstruktur und der Qualität des Unterrichts sowie die Förderung des Lernens an einem gemeinsamen Gegenstand (Moser Opitz 2014, 
S. 53). Darüber hinaus wurde die Auswahl der Konzepte so getroffen, dass sie die Bandbreite der inneren Differenzierung in der Mathematikdidaktik abbilden.

\subsubsection{Lernumgebungen für den inklusiven Mathematikunterricht (Häsel-Weide} 2015, 2016a, 2016b, 2017)

Zur Gestaltung eines inklusiven Mathematikunterrichts adaptiert Häsel-Weide (2015, 2016a, 2016b, 2017) sogenannte substantielle Lernumgebungen und stellt dar, wie diese mit Blick auf Kinder mit dem SPU LE in den Unterricht integriert werden können. Traditionellen substantiellen Lernumgebungen liegen Aufgabenformate zu Grunde, die eine natürliche Differenzierung ermöglichen. Sie sind an einem gemeinsamen Gegenstand ausgerichtet und orientieren sich inhaltlich an fundamentalen Ideen der Mathematik (für die Grundschule werden hierfür häufig die Kataloge von Winter (2001) oder Wittmann (1998) herangezogen). Unter einem Aufgabenformat können flexible, große Aufgaben verstanden werden, die aus mehreren Teilaufgaben und Arbeitsanweisungen bestehen und bezüglich der innermathematischen oder sachbezogenen Struktur miteinander verbunden sind (Hirt und Wälti 2010). Um an die individuellen Kompetenzen der Kinder anschließen zu können, verfügen die Lernumgebungen für einen inklusiven Unterricht über eine niedrige Einstiegsschwelle und sind darüber hinaus ausreichend reichhaltig gestaltet. Zudem liefern sie Anregungen für kooperatives Handeln und für die Lernenden mit besonderem Unterstützungsbedarf genügend Gelegenheit zur Erarbeitung des Basisstoffs. Durch Parallelisierungen, sprich der Bearbeitung analoger Aufgaben in unterschiedlichen Zahlenräumen und unterrichtsintegrierten Fördermaßnahmen, kann das Arbeiten auf individuellen Niveaus zusätzlich unterstützt werden (Häsel-Weide 2016b, S. 12f.).

Häsel-Weide (2016b, S. 20f.) hebt hervor, dass die Orientierung an einem gemeinsamen Gegenstand eine Begrenzung erfordert, damit ein inhaltlicher Austausch aller Lernenden über die gemeinsame Idee stattfinden kann. Andererseits muss der Lerngegenstand hinreichend offen für die individuellen Möglichkeiten der Kinder sein. Um einen für alle Kinder lernförderlichen guten inklusiven Unterricht zu ermöglichen, erscheint die Realisierung unterschiedlicher Lernumgebungen mit unterschiedlicher Ausrichtung von Offenheit und Kooperation in Verbindung mit einem Wechsel von Phasen des gemeinsamen und individuellen Lernens im Unterricht sinnvoll.

\subsubsection{Mathe inklusiv mit PIKAS (DZLM 2015)}

Ein weiteres Konzept zur Gestaltung inklusiven Mathematikunterrichts entwickelt das Projekt Mathematik inklusiv mit PIKAS seit 2015 (DZLM 2015). Das Projekt verfolgt das Ziel, Lehrkräfte der Primarstufe bei der Planung, Durchführung und Reflexion inklusiven Mathematikunterrichts zu unterstützen. In Form einer Webseite werden Unterrichtsideen, Materialien, Informationstexte sowie mathematikdidaktische Theorien praxisnah aufgearbeitet. Unter der Rubrik Leitideen werden zunächst inhaltsübergreifende Aspekte guten Mathematikunterrichts, wie das Adaptieren von Aufgaben, das diagnosegeleitete Fördern, effektives Üben und das Anregen eines gemeinsamen Austausches auf inklusive Lernsituationen übertragen und Umset- 
zungsmöglichkeiten für die Praxis an konkreten Beispielen veranschaulicht. Unter der Rubrik Inhalte werden zu bisher sechs zentralen Themen aus den Inhaltsbereichen Zahlen und Operationen und Größen und Messen grundlegende Aufgabenstellungen sowie verschiedene Adaptionen dieser Aufgaben konkretisiert. Durch die Adaptionen wird die inhaltliche Bandbreite einer Aufgabe aufgezeigt, die eine Differenzierung sowohl für Kinder mit besonderen Kompetenzen als auch für Kinder mit einem SPU ermöglicht. Zu den Aufgabenstellungen erhalten Lehrkräfte Informationen über die grundlegende Bedeutung des Inhaltes, die elementaren Lernziele sowie Materialien zur Durchführung der Unterrichtsvorschläge. Anhand einzelner Unterrichtssequenzen wird dargestellt, wie gemeinsame Lernsituationen gestaltet und dabei mit einer gemeinsamen Aufgabenstellung verschiedene Lernziele erreicht werden können. Die Rubrik Förderschwerpunkte liefert Lehrkräften zusätzlich spezifische Unterstützungsmaßnahmen zu den sieben verschiedenen SPU. Die Unterrichtsmaterialien von Mathe inklusiv haben zwar einen exemplarischen Charakter, der Grundgedanke ist jedoch, Lehrkräfte durch die beispielhaften Konkretisierungen für die Grundzüge eines guten inklusiven Mathematikunterrichts zu sensibilisieren und für eine Übertragung auf weitere Inhalte zu professionalisieren.

\subsubsection{Das Zahlenbuch (Wittmann et al. 2017a, 2017b, 2018, 2019)}

Viele traditionelle Mathematiklehrwerke haben in den letzten Jahren auf die größer werdende Heterogenität im Klassenzimmer reagiert und ihre Konzepte mit Hilfe spezieller Förderhefte auf inklusives Unterrichten angepasst. Beispielhaft stellen wir hier das Lehrwerk Das Zahlenbuch in der Neubearbeitung von Nührenbörger und Schwarzkopf (Wittmann et al. 2017a, 2017b, 2018, 2019) vor, die entsprechend der Kriterien von Moser Opitz (2014) ein Konzept für inklusiven Mathematikunterricht in den Klassen 1 bis 4 vorlegen. Das Lehrwerk unterteilt die Inhalte der Grundschulmathematik in Themenblöcke, die sich in jedem Schuljahr in ähnlicher Form wiederholen und damit entsprechend der fundamentalen Ideen spiralcurricular bearbeitet werden können. Zu jedem Themenblock werden Basiskompetenzen ausgewiesen, die als Grundlage für alle Kinder verbindlich sind. Im Sinne einer natürlichen Differenzierung bauen weitere Lernangebote auf diesen Basiskompetenzen auf. Durch niedrigschwellige thematische Einstiege, durchgängige Aufforderungen zum Sprechen über die mathematischen Inhalte sowie weiterführenden Aufgaben, die je nach Lernausgangslage in unterschiedlichem Umfang und Tiefe bearbeitet werden können, wird ein gemeinsames Lernen auf individuellem Niveau ermöglicht. Für weitere, durch die Lehrkraft vorgegebene, Differenzierungen liegen Förderkommentare für die SPU LE und SQ sowie Förderhefte und differenzierte Lernstandskontrollen vor. Der Förderkommentar Lernen (Häsel-Weide und Nührenbörger 2017a, 2017b, 2018, 2019) beinhaltet Ergänzungen zu ausgewählten Schulbuchseiten, um Kinder bei der Bewältigung der mathematischen Grundlagen zu unterstützen. Das Förderheft bietet passend zu den Seiten des Schulbuchs adaptierte Aufgaben an. Im Förderkommentar Sprache (Götze und Hang 2017a, 2017b, 2018, 2019) werden zu zentralen Themenschwerpunkten sprachliche Anforderungen sowie mögliche individuelle sprachliche Hürden aufgezeigt. Anhand der zentralen Schwerpunkte Fachwortschatz aufbauen, Sprachverständnis sichern und mathema- 
tische Kommunikation fördern werden Vorschläge dargelegt, wie spezifische Aufgabenstellungen des Schülerbuchs sprachsensibel angereichert werden können (Götze und Hang 2017a, S. 8 f.).

\subsection{Zusammenfassung}

Die dargestellte Auswahl an Konzepten liefert einen Überblick über unterschiedliche Differenzierungsmöglichkeiten, die die Mathematikdidaktik innerhalb des gemeinsamen Lernens in heterogenen Lerngruppen bietet. Während in den Lernumgebungen von Häsel-Weide eine natürliche Differenzierung vom Kind aus erfolgt, werden in Mathe inklusiv mit PIKAS durch Adaption einer grundlegenden Aufgabe paralleldifferenzierende Aufgaben geschaffen, die den Kindern von der Lehrkraft zugewiesen werden. Auch eine Kombination von natürlicher Differenzierung und Aufgabenadaptionen scheint ein möglicher Weg zur individuellen Förderung zu sein, wie Das Zahlenbuch zeigt. Die mathematikdidaktischen Konzepte entsprechen allesamt den genannten Kriterien einer inklusiven Didaktik (Moser Opitz 2014). Sie sind auf das Lernen an einen gemeinsamen Gegenstand ausgerichtet, weisen Differenzierungsmaßnahmen aus und orientieren sich an fundamentalen Ideen der Mathematik. Es mangelt bislang jedoch an Evaluationen, inwieweit die Konzepte in der Praxis umsetzbar und für den Lernerfolg aller Kinder effektiv sind. Darüber hinaus finden sich nur im Konzept Mathe inklusiv mit PIKAS Vorschläge für die unterschiedlichen Unterstützungsbedarfe, insgesamt nehmen die Konzepte schwerpunktmäßig die Bandbreite der Kinder ohne SPU und die SPU LE sowie SQ in den Fokus. Bezogen auf die weitere Entwicklung von mathematikdidaktischen Konzepten wäre folglich eine Ausrichtung auf die verschiedenen Unterstützungsbedarfe wünschenswert.

\section{Empirische Befunde zur aktuellen Praxis inklusiven Mathematikunterrichts in der Grundschule}

Ausgehend vom Stand der Entwicklung mathematikdidaktischer Konzeptionen und konkreter Konzepte für einen inklusiven Unterricht interessiert es, wie Lehrkräfte die Gestaltung eines inklusiven Unterrichts bezogen auf das Fach einschätzen und welche Erkenntnisse bereits evaluierte Konzepte liefern können. Da es bislang nur wenige aktuelle Studien gibt, die sich diesen Forschungsinteressen widmen, liegt der Fokus im Folgenden auf zwei ausgewählten Studien zu den Erfahrungen von Lehrkräften sowie auf zwei evaluierten mathematikdidaktischen Konzepten, die in der Schweiz entwickelt wurden. 


\subsection{Praxiseindrücke und Erfahrungen von Lehrkräften zu gemeinsamem Lernen}

\subsubsection{Erfahrungen, Perspektiven und Herausforderungen von Lehrkräften (Korff 2015, D)}

Vor dem Hintergrund dessen, dass Lehrkräfte immer wieder auf besondere Herausforderungen des Fachs Mathematik bezogen auf einen inklusiven Unterricht verweisen, führte Korff (2015) eine qualitativ angelegte Interviewstudie $(n=14) \mathrm{zu}$ Erfahrungen und Perspektiven von Lehrkräften für Grundschul- und Sonderpädagogik durch. Die Ergebnisse legen dar, dass die Gestaltung von gemeinsamen Lernsituationen im Fach Mathematik für alle Lehrkräfte eine besondere Herausforderung darstellt. Lehrkräfte mit wenig Erfahrung im inklusiven Mathematikunterricht vertreten darüber hinaus die Ansicht, dass Mathematik schwieriger gemeinsam zu unterrichten ist als andere Fächer. Mit Blick auf die unterschiedlichen mathematischen Inhaltsbereiche sind die Bereiche Geometrie, Größen und Sachrechnen im Sinne des mit- und voneinander Lernens wiederum einfacher zu gestalten als die Arithmetik. Die arithmetischen Inhalte sind nach Aussage der Lehrkräfte zu hierarchisch aufgebaut, um gemeinsame Themen zu finden. Darüber hinaus empfinden sie Zahlen und Operationen als die wesentlichen Inhalte der Arithmetik als (zu) abstrakt, um eine handlungs- und materialorientierte Bearbeitungen für alle Kinder ermöglichen zu können. Daher werden für die Umsetzung arithmetischer Inhalte eher individuelle, ,symbol- und arbeitsblattorientierte“ (ebd., S. 204) Lernsituationen bevorzugt, obwohl die Lehrkräfte herausstellen, dass grundsätzlich ein mit- und voneinander Lernen durch das Vorhandensein von Anschaulichkeit, Handlungs- und Materialorientierung in allen Fächern begünstigt werden kann.

\subsubsection{Fallstudien zu gemeinsamen Lernsituationen (Oechsle 2020, D)}

Ausgehend von der Tatsache, dass sich der inklusive Unterricht im Fach Mathematik in den letzten Jahren weiterentwickelt hat und verschiedene neue Lehrwerke und Unterrichtsvorschläge konzipiert wurden, setzte sich die Studie mit der Frage auseinander, wie Lehrkräfte für Grundschul- und Sonderpädagogik einen inklusiven Mathematikunterricht gegenwärtig gestalten und welche Überzeugungen hinter der Unterrichtsgestaltung stehen (Oechsle 2020, S. 2ff.). Die Ergebnisse einer Interviewstudie $(n=19)$ und einer Videostudie $(n=3)$ legen dar, dass ein unterschiedliches Verständnis davon besteht, was gemeinsame Lernsituationen ausmachen (ebd., S. $218 \mathrm{ff}$.). Lehrkräfte nehmen die Gestaltung inklusiven Mathematikunterrichts insbesondere dann als Herausforderung wahr, wenn große Leistungsunterschiede innerhalb einer Klasse bestehen und wenn die Sorge aufkommt, den Kindern ohne SPU nicht gerecht werden zu können (ebd., S. 216f.). Die Aussagen einzelner Lehrkräfte verdeutlichen, dass sich arithmetische Inhalte, aufgrund ihres hierarchischen Aufbaus sowie fehlender Veranschaulichungsmöglichkeiten, scheinbar weniger für gemeinsame Lernsituationen eignen als beispielsweise geometrische Inhalte. Darüber hinaus wird inklusiver Unterricht mit Kindern mit dem SPU GG als besonders anspruchsvoll betrachtet, da es an Erfahrungen und Unterrichtsideen mangelt. Die 
Ergebnisse weisen darauf hin, dass die Entwicklung und Evaluation geeigneter Lehrwerke und Unterrichtsmaterialien sowie die Überarbeitung bzw. Parallelisierung der unterschiedlichen Bildungspläne mit dem Ziel einer gegenseitigen Anschlussfähigkeit notwendig ist, um Lehrkräfte bei der Gestaltung inklusiven Mathematikunterrichts zu unterstützen (ebd., S. 225 ff.).

\subsection{Entwicklung und Evaluation inklusiven Unterrichts}

\subsubsection{Produktives Rechnen im integrativen Mathematikunterricht (Stöckli et al. 2014, $\mathrm{CH}$ )}

Im Rahmen des schweizerischen Förderkonzepts PRiMa (Produktives Rechnen im integrativen Mathematikunterricht) wurden Unterrichtseinheiten für ein drittes Schuljahr zur Unterstützung rechenschwacher Kinder im arithmetischen Bereich entwickelt und evaluiert. Neben Anregungen und Ergänzungen zu den verwendeten Schulbüchern wurden paralleldifferenzierende Aufgaben entwickelt. Grundgedanke war, dass die Kinder im Unterricht zunächst differenziert nach individuellen Voraussetzungen an einem gemeinsamen mathematischen Gegenstand und anschließend individuell an Aufgaben zur jeweiligen Unterrichtseinheit arbeiten. Ein besonderer Fokus wurde auf die Interaktion der Lernenden und eine gemeinsame Reflexion der Unterrichtsinhalte gelegt (Stöckli et al. 2014). Die Wirksamkeit der unterrichtsintegrierten Fördermaßnahmen wurde anhand von zwei Interventionsgruppen und einer Kontrollgruppe erhoben. Die Ergebnisse konnten zeigen, dass die Fördermaßnahmen bezogen auf die Leistungsentwicklung der Kinder nur in einer der Interventionsgruppen effektiv waren. Mögliche Erklärungen für die Ergebnisse können in der großen Heterogenität inklusiver Klassen, den unterschiedlichen Rahmenbedingungen und der Beteiligung mehrerer Lehrkräfte am Unterrichtsgeschehen gesehen werden (Stöckli 2019).

\subsubsection{Mathematische Förderung von Kindern mit dem SPU GG (Schnepel 2019, $\mathrm{CH})$}

Ein Konzept zur Gestaltung inklusiven Mathematikunterrichts für Kinder mit dem SPU GG wurde von Schnepel (2019) entwickelt und evaluiert. Neben dem Ziel, dass alle Kinder mathematische Inhalte ,ganzheitlich in fachlich sinnvollen Zusammenhängen strukturiert auf ihrem jeweiligen Niveau“ bearbeiten können (ebd., S. 129), soll in gleicher Weise an einem gemeinsamen Lerninhalt gearbeitet werden. Der inhaltliche Fokus des Konzepts liegt auf der Förderung der Mengen-Zahlen-Kompetenzen, da diese für Kinder mit dem SPU GG häufig mit Schwierigkeiten verbunden ist. Gemeinsame Lernsituationen finden in den Einstiegs- und Reflexionsphasen einer Unterrichtsstunde statt. Zwischen den gemeinsamen Unterrichtssequenzen sind Arbeitsphasen vorgesehen, in denen die Kinder ausgehend von ihren individuellen Lernniveaus selbständig oder mit Mitschüler*innen gemeinsam an differenzierten Materialien arbeiten (ebd., S. $130 \mathrm{ff}$.). Damit die Kinder neben den individuellen Arbeitsphasen in einen mathematischen Austausch treten können, wurde den Inhalten im Mathematikbuch als sogenannte Anknüpfungspunkte passende Mengen- 
Zahlen-Kompetenzen zugeordnet, die den gemeinsamen Lerninhalt bilden. $\mathrm{Zu}$ allen Anknüpfungspunkten wurden Förderideen entwickelt, um vielfältige Differenzierungsmöglichkeiten für alle Lernenden zu schaffen (ebd., S. 133). Die Studie konnte keine Unterschiede in der mathematischen Entwicklung der Kinder mit und ohne SPU GG zwischen Interventions- und Kontrollgruppe offenlegen. Dass wünschenswerte Interventionseffekte ausblieben, kann auf verschiedene Faktoren wie die Implementierung der Intervention in den Unterricht durch die jeweilige Lehrkraft, den Interventionszeitraum und die Kontrollgruppe zurückgeführt werden (ebd., S. $196 \mathrm{ff}$.$) .$

\subsection{Zusammenfassung}

Empirische Befunde zum aktuellen inklusiven Mathematikunterricht zeigen, dass die Gestaltung gemeinsamen mathematischen Lernens von Lehrkräften als herausfordernd wahrgenommen wird. Dies trifft vor allem dann zu, wenn die Leistungsunterschiede zwischen den Kindern sehr groß sind, was bei der Inklusion von Kindern mit dem SPU GG zwangsläufig der Fall ist. Insbesondere für die arithmetischen Inhalte ist ein mit- und voneinander Lernen, im Gegensatz zu den anderen mathematischen Inhaltsbereichen, schwieriger zu gestalten. Die Lehrkräfte erklären die Herausforderungen im Bereich der Arithmetik mit dem hierarchischen Aufbau und der Abstraktheit der Inhalte, wodurch diese für gemeinsame Lernsituationen und eine handlungs- und materialbasierte Zugangsweise ungeeignet wirken. Aus mathematikdidaktischer Perspektive scheint die besondere Herausforderung für Lehrkräfte die Identifizierung fundamentaler Ideen der Arithmetik, die auf unterschiedlichen Niveaus bearbeitet werden können, sowie die Gestaltung unterschiedlicher Zugangsweisen zu arithmetischen Inhalten zu sein. Die Konzepte aus der Schweiz konkretisieren die konzeptionellen Überlegungen der Mathematikdidaktik und entsprechen dem von Lehrkräften geäußerten Desiderat zu zeigen, wie gemeinsames Lernen und individuelle Förderung im Arithmetikunterricht miteinander verbunden werden kann. Die Ergebnisse der Evaluation entsprechen zwar nicht den erhofften Annahmen im Hinblick auf eine positivere mathematische Entwicklung der Kinder, sie bieten jedoch Ansätze für weitere didaktische Entwicklungen.

\section{Diskussion und Ausblick}

Wenn das langfristige Ziel für alle Kinder eine Teilhabe - und nicht nur eine Teilnahme - an der Gesellschaft ist, ist es aus mathematikdidaktischer Perspektive wichtig, dass fachliches Lernen im inklusiven Mathematikunterricht für alle Kinder gewährleistet wird. Die referierten Studien (Abschn. 2 und 4.2) stützen diese Forderung: Kinder mit und ohne SPU lernen in inklusiven Settings genauso gut Mathematik wie in separativen Settings, zum Teil ergeben sich sogar positive Effekte. Aus den empirischen Ergebnissen, zusammen mit den in den letzten Jahren in der Mathematikdidaktik entwickelten Konzepten für gemeinsames mathematisches Lernen (Abschn. 3.2 und 4.2) schließen wir, dass ein inklusiver Mathematikunterricht aus fachlicher Sicht möglich ist und auch effektiv sein kann. 
Bei genauerer Betrachtung stehen die Studienergebnisse (Abschn. 2) jedoch im Widerspruch zu der von Lehrkräften berichteten Praxis inklusiven Unterrichts (Abschn. 4.1). Lehrkräfte nennen die Gestaltung gemeinsamer Lernsituationen für arithmetische Inhalte als besondere Herausforderung, weshalb im Arithmetikunterricht scheinbar eher individuelles statt gemeinsames Lernen praktiziert wird. Obwohl also scheinbar keine gemeinsamen Lernsituationen stattfinden, können bei den arithmetischen Kompetenzen, die die meisten Studien ausschließlich testeten, dennoch neutrale bis positive Effekte des inklusiven Matheunterrichts auf die kindliche Lernentwicklung festgestellt werden. Es lässt sich daher vermuten, dass in einem Arithmetikunterricht, der auf mit- und voneinander Lernen im Sinne eines inhaltlichen Austauschs zwischen den Kindern angelegt ist, noch positivere Effekte auf die Lernentwicklung der Kinder zu erwarten sind (vgl. auch Freeman und Alkin 2000; Abschn. 2.3). Die referierten Schweizer Studien (Abschn. 4.2) zeigen jedoch, dass Effekte trotz fachlich fundierter Unterrichtsplanung nicht einfach zu erzeugen sind. Als mögliche Erklärung ist unter anderem anzunehmen, dass sich ein messbarer Effekt gemeinsamen Lernens auf die arithmetische Entwicklung von Kindern erst über einen längeren Zeitraum zeigt (vgl. Abschn. 2.3). Aufgrund der Berichte von Lehrkräften, dass die Gestaltung gemeinsamer Lernsituationen für z. B. geometrische Inhalte einfacher gelingt und der Tatsache, dass sich mathematisches Lernen nicht nur auf die Arithmetik beschränkt, gilt es in zukünftigen Forschungsarbeiten auch die mathematische Lernentwicklung in den anderen Inhaltsbereichen des Fachs in den Blick zu nehmen. Inwiefern sich das mit- und voneinander Lernen am gemeinsamen Gegenstand, das auch durch die allgemeinen Kompetenzen gestützt wird, auf das emotional-soziale Wohlbefinden der Kinder einer Klasse auswirkt, ist ebenfalls noch zu untersuchen (vgl. Peter-Koop und Lüken 2017).

Die bisherigen mathematikdidaktischen Entwicklungen zur Gestaltung eines inklusiven Unterrichts orientieren sich auf theoretischer sowie auf konzeptioneller Ebene zum einen an dem Grundgedanken, dass Lernen an einem gemeinsamen Gegenstand stattfinden sollte und zum anderen an der natürlichen Differenzierung und fundamentalen Ideen des Fachs (Abschn. 3.1). Die aufgezeigten Konzepte gestalten ein gemeinsames Lernen durch unterschiedliche Möglichkeiten zur Differenzierung der fachlichen Inhalte aus und legen den Fokus auf Kinder mit dem SPU LE sowie SQ (Abschn. 3.2). Weitere Forschungsprojekte sollten allerdings auch die SPU in den Blick nehmen, zu denen bislang nur wenige Überlegungen vorliegen. In allen Konzepten wird deutlich, dass ein Spannungsfeld zwischen gemeinsam lernen und effektiv fördern grundsätzlich bestehen bleibt und ein Wechsel von Phasen des gemeinsamen Lernens und individuellen Bearbeitungen sinnvoll ist. Die Ergebnisse aus Abschn. 4.1 zeigen jedoch, dass Lehrkräfte Schwierigkeiten haben, für arithmetische Inhalte gemeinsame Lernsituationen zu initiieren. Die Begründungen der Lehrkräfte zum einen über den hierarchischen Aufbau der Inhalte, der scheinbar gemeinsame Themen verhindert, interpretieren wir als Schwierigkeit, fundamentale Ideen der Grundschularithmetik zu identifizieren. Fundamentale Ideen zeigen mathematische Inhalte auf, die für die Entwicklung aller Kinder bedeutsam sind und die spiralcurricular auf unterschiedlichen Ebenen bearbeitet werden können (vgl. Abschn. 3.1). An dieser Stelle besteht weiterer Entwicklungsbedarf auf Seiten der Mathematikdidaktik, da bereits ausgearbeitete Konzepte, die sich an fundamentalen Ideen orientieren 
und sich passgenau dem von Lehrkräften als schwierig empfundenen arithmetischen Bereich widmen, bislang nur einen exemplarischen Charakter besitzen. Zum anderen scheint der aktuell im Unterricht vorherrschende symbolorientierte Zugang auf Zahlen- und Operationsebene für Kinder ohne SPU ein gemeinsames Lernen zu verhindern. Aus mathedidaktischer Perspektive sind Material- bzw. Handlungsorientierung und abstrakte Mathematik keine Gegensätze. Vielmehr können Handlungen an Alltagsobjekten und am didaktischen Material als Grundlage für ein Verständnis mathematischer Begriffe betrachtet werden (Bruner 1974; vom Hofe 1995). Hier gilt es, Lehrkräfte gezielt zu professionalisieren, wie inhaltlich-abstrakte Ideen der Mathematik auch in verschiedenen Darstellungsformen thematisiert werden können (vgl. auch Korff 2015, S. 237). Das Ergebnis, dass inklusions-erfahrene Lehrkräfte das Fach Mathematik nicht schwieriger inklusiv zu unterrichten finden als andere Fächer, könnte außerdem auf einen Professionalisierungsprozess mit zunehmender Erfahrung hinweisen.

Es kann festgehalten werden, dass die Gestaltung inklusiven Mathematikunterrichts komplex ist und dass nach wie vor Forschungsbedarf im Bereich der Unterrichtsentwicklung besteht, damit alle Kinder bestmöglich mit- und voneinander lernen können.

Funding Open Access funding enabled and organized by Projekt DEAL.

Open Access Dieser Artikel wird unter der Creative Commons Namensnennung 4.0 International Lizenz veröffentlicht, welche die Nutzung, Vervielfältigung, Bearbeitung, Verbreitung und Wiedergabe in jeglichem Medium und Format erlaubt, sofern Sie den/die ursprünglichen Autor(en) und die Quelle ordnungsgemäß nennen, einen Link zur Creative Commons Lizenz beifügen und angeben, ob Änderungen vorgenommen wurden.

Die in diesem Artikel enthaltenen Bilder und sonstiges Drittmaterial unterliegen ebenfalls der genannten Creative Commons Lizenz, sofern sich aus der Abbildungslegende nichts anderes ergibt. Sofern das betreffende Material nicht unter der genannten Creative Commons Lizenz steht und die betreffende Handlung nicht nach gesetzlichen Vorschriften erlaubt ist, ist für die oben aufgeführten Weiterverwendungen des Materials die Einwilligung des jeweiligen Rechteinhabers einzuholen.

Weitere Details zur Lizenz entnehmen Sie bitte der Lizenzinformation auf http://creativecommons.org/ licenses/by/4.0/deed.de.

\section{Literatur}

Affleck, J.Q., Madge, S., Adams, A., \& Lowenbraun, S. (1988). Integrated classroom versus resource model: academic viability and effectiveness. Exceptional Children, 54(4), 339-348.

Bear, G. G., \& Proctor, W. A. (1990). Impact of a full-time integrated program on the achievement of nonhandicapped and mildly handicapped children. Journal of Exceptionality, 1(4), 227-238.

Bless, G. (1995). Zur Wirksamkeit der Integration: Forschungsüberblick, praktische Umsetzung einer integrativen Schulform, Untersuchungen zum Lernfortschritt. Bern: Haupt.

Bruner, J.S. (1974). Entwurf einer Unterrichtstheorie. Berlin: Berlin-Verlag.

Budoff, M., \& Gottlieb, J. (1976). Special-class EMR children mainstreamed: a study of an aptitude (learning potential) X treatment interaction. American Journal of Mental Deficiency, 81(1), 1-11.

Cole, C. M., Waldron, N., \& Maijd, M. (2004). Academic progress of students across inclusive and traditional settings. Mental Retardation, 42(2), 136-144.

DZLM: Deutsches Zentrum für Lehrerbildung Mathematik (2015). Mathe inklusiv mit PIKAS. https:// pikas-mi.dzlm.de. Zugegriffen: 06.05.2020.

Fetzer, M. (2019). Inklusiver Mathematikunterricht. Baltmannsweiler: Schneider. 
Feuser, G. (1998). Gemeinsames Lernen am Gemeinsamen Gegenstand. Didaktisches Fundamentum einer Allgemeinen (integrativen) Pädagogik. In A. Hildeschmidt \& I. Schnell (Hrsg.), Integrationspädagogik. Auf dem Weg zu einer Schule für alle (S. 19-36). Weinheim: Juventa.

Freeman, S. F., \& Alkin, M.C. (2000). Academic and social attainments of children with mental retardation in general education and special education settings. Remedial and Special Education, 21(1), 3-18.

Götze, D., \& Hang, E. (2017a). Das Zahlenbuch. Förderkommentar Sprache zum 1. Schuljahr. Stuttgart: Klett.

Götze, D., \& Hang, E. (2017b). Das Zahlenbuch. Förderkommentar Sprache zum 2. Schuljahr. Stuttgart: Klett.

Götze, D., \& Hang, E. (2018). Das Zahlenbuch. Förderkommentar Sprache zum 3. Schuljahr. Stuttgart: Klett.

Götze, D., \& Hang, E. (2019). Das Zahlenbuch. Förderkommentar Sprache zum 4. Schuljahr. Stuttgart: Klett.

Haeberlin, U., Bless, G., Moser, U., \& Klaghofer, R. (1999). Die Integration von Lernbehinderten: Versuche, Theorien, Forschungen, Enttäuschungen, Hoffnungen. Bern: Haupt.

Häsel-Weide, U. (2015). Gemeinsam Mathematik lernen. Überlegungen für den inklusiven Mathematikunterricht. Grundschule aktuell, 130, 3-7.

Häsel-Weide, U. (2016a). Gemeinsam ordnen - Gemeinsam lernen. Mathematische Strukturen sichtbar machen. Grundschulunterricht Mathematik, 1, 30-33.

Häsel-Weide, U. (2016b). Mathematik gemeinsam lernen - Lernumgebungen für den inklusiven Mathematikunterricht. In A.S. Steinweg (Hrsg.), Inklusiver Mathematikunterricht - Mathematiklernen in ausgewählten Förderschwerpunkten. Tagungsband des AK Grundschule in der GDM 2016. (S. 9-24). Magdeburg: Dokupoint.

Häsel-Weide, U. (2017). Inklusiven Mathematikunterricht gestalten. Anforderungen an die Lehrerausbildung. In J. Leuders, T. Leuders, S. Prediger \& S. Ruwisch (Hrsg.), Mit Heterogenität im Mathematikunterricht umgehen lernen: Konzepte und Perspektiven für eine zentrale Anforderung an die Lehrerbildung (S. 17-28). Wiesbaden: Springer.

Häsel-Weide, U., \& Nührenbörger, M. (2015). Aufgabenformate für einen inklusiven Arithmetikunterricht. In A. Peter-Koop, T. Rottmann \& M. M. Lüken (Hrsg.), Inklusiver Mathematikunterricht in der Grundschule (S. 58-74). Offenburg: Mildenberger.

Häsel-Weide, U. \& Nührenbörger, M. (2017a). Das Zahlenbuch. Förderkommentar Lernen zum 1. Schuljahr. Stuttgart: Klett.

Häsel-Weide, U. \& Nührenbörger, M. (2017b). Das Zahlenbuch. Förderkommentar Lernen zum 2. Schuljahr. Stuttgart: Klett.

Häsel-Weide, U., \& Nührenbörger, M. (2017c). Grundzüge des inklusiven Mathematikunterrichts. Mit allen Kindern rechnen. In U. Häsel-Weide \& M. Nührenbörger (Hrsg.), Gemeinsam Mathematik lernen - Mit allen Kindern rechnen (S. 8-21). Frankfurt a. M.: Grundschulverband e. V.

Häsel-Weide, U. \& Nührenbörger, M. (2018). Das Zahlenbuch. Förderkommentar Lernen zum 3. Schuljahr. Stuttgart: Klett.

Häsel-Weide, U., \& Nührenbörger, M. (2019). Das Zahlenbuch. Förderkommentar Lernen zum 4. Schuljahr. Stuttgart: Klett.

Hirt, U., \& Wälti, B. (2010). Lernumgebungen im Mathematikunterricht. Natürliche Differenzierung für Rechenschwache bis Hochbegabte. Seelze: Klett.

vom Hofe, R. (1995). Grundvorstellungen mathematischer Inhalte. Heidelberg: Spektrum. Akademischer Verlag.

Huber, K. D., Rosenfeld, J. G., \& Fiorello, C. A. (2001). The differential impact of inclusion and inclusive practices on high, average, and low achieving general education students. Psychology in the Schools, 38(6), 497-504.

Hunt, P., Staub, D., Alwell, M., \& Goetz, L. (1994). Achievement by all students within the context of cooperative learning groups. Journal of the Association for Persons with Severe Handicaps, 19(4), 290-301.

Käpnick, F. (Hrsg.). (2016). Verschieden verschiedene Kinder: Inklusives Fördern im Mathematikunterricht der Grundschule. Seelze: Klett.

Karsten, S., Peetsma, T., Roeleveld, J., \& Vergeer, M. (2001). The Dutch policy of integration put to the test: differences in academic and psychosocial development of pupils in special and mainstream education. European Journal of Special Needs Education, 16(3), 193-205.

KMK: Kultusministerkonferenz (2004). Bildungsstandards im Fach Mathematik für die Primarstufe. https://www.kmk.org/fileadmin/Dateien/veroeffentlichungen_beschluesse/2004/2004_10_15Bildungsstandards-Mathe-Primar.pdf. Zugegriffen: 19.05.2020. 
Kocaj, A., Kuhl, P., Kroth, A.J., Pant, H. A., \& Stanat, P. (2014). Wo lernen Kinder mit sonderpädagogischem Förderbedarf besser? Ein Vergleich schulischer Kompetenzen zwischen Regel- und Förderschulen in der Primarstufe. Kölner Zeitschrift für Soziologie und Sozialpsychologie, 66(2), 165-191.

Korff, N. (2015). Inklusiver Mathematikunterricht in der Primarstufe. Baltmannsweiler: Schneider.

Krauthausen, G., \& Scherer, P. (2014). Natürliche Differenzierung im Mathematikunterricht: Konzepte und Praxisbeispiele aus der Grundschule. Seelze: Klett.

Leuders, J. (2016). Inklusives Mathematiklernen bei Sehbeeinträchtigung und Blindheit - Herausforderungen und Konzepte. In A.S. Steinweg (Hrsg.), Inklusiver Mathematikunterricht - Mathematiklernen in ausgewählten Förderschwerpunkten. Tagungsband des AK Grundschule in der GDM 2016. (S. 41-56). Magdeburg: Dokupoint.

McDonnell, J., Thorson, N., Disher, S., Mathot-Buckner, C., Mendel, J., \& Ray, L. (2003). The achievement of students with developmental disabilities and their peers without disabilities in inclusive settings: an exploratory study. Education and Treatment of Children, 26(3), 224-236.

Moser Opitz, E. (2014). Inklusive Didaktik im Spannungsfeld von gemeinsamem Lernen und effektiver Förderung. Ein Forschungsüberblick und eine Analyse von didaktischen Konzeptionen für inklusiven Unterricht. Jahrbuch für allgemeine Didaktik, 4(3), 52-68.

Moser Opitz, E. (2016). Inklusiver Mathematikunterricht - Auch für Schülerinnen und Schüler mit dem Förderschwerpunkt Geistige. In Entwicklung (FGE). In A. S. Steinweg (Hrsg.), Inklusiver Mathematikunterricht - Mathematiklernen in ausgewählten Förderschwerpunkten. Tagungsband des AK Grundschule in der GDM 2016. (S. 57-60). Magdeburg: Dokupoint.

MSB NRW: Ministerium für Schule und Bildung des Landes Nordrhein-Westfalen Lexikon der Inklusion. https://www.schulministerium.nrw.de/docs/Schulsystem/Inklusion/Eltern/2-Lexikon-der-Inklusion/ index.html. Zugegriffen: 14.05.2020.

Oechsle, U. (2020). Mathematikunterricht im Kontext von Inklusion. Fallstudien zu gemeinsamen Lernsituationen. Wiesbaden: Springer Spektrum.

Peetsma, T., Vergeer, M., Roeleveld, J., \& Karsten, S. (2001). Inclusion in education: comparing pupils' development in special and regular education. Educational Review, 53(2), 125-135.

Peter-Koop, A., \& Lüken, M. (2017). Early years mathematics learning - Comparing traditional and inclusive classroom settings. In T. Dooley \& G. Gueudet (Hrsg.), Proceedings of the Tenth Congress of the European Society for Research in Mathematics Education. CERME 10 (S. 1942-1943). Dublin, Ireland: Institute of Education, Dublin City University; ERME.

Rathgeb-Schnierer, E., \& Feindt, A. (2014). 24 Aufgaben für 24 Kinder oder eine Aufgabe für alle? Die Grundschulzeitschrift, 28(271), 30-35.

Ratz, C. (2017). Inklusive Didaktik für den Förderschwerpunkt Geistige Entwicklung. In E. Fischer \& C. Ratz (Hrsg.), Inklusion - Chancen und Herausforderungen für Menschen mit geistiger Behinderung (S. 172-191). Weinheim: Beltz.

Ruijs, N. M., \& Peetsma, T. T.D. (2009). Effects of inclusion on students with and without special educational needs reviewed. Educational Research Review, 4(2), 67-79.

Saint-Laurent, L., Dionne, J., Giasson, J., Royer, E., Simard, C., \& Pierard, B. (1998). Academic achievement effects of an in-class service model on students with and without disabilities. Exceptional Children, 64(2), 239-253.

Scherer, P. (2017). Gemeinsames Lernen oder Einzelförderung? - Grenzen und Möglichkeiten eines inklusiven Mathematikunterrichts. In F. Hellmich (Hrsg.), Inklusiver Unterricht in der Grundschule (S. 194-212). Stuttgart: Kohlhammer.

Schindler, M. (2017). Inklusiver Mathematikunterricht am gemeinsamen Gegenstand. Mathematik lehren, 201, 6-10.

Schnepel, S. (2019). Mathematische Förderung von Kindern mit einer intellektuellen Beeinträchtigung. Münster: Waxmann.

Sermier Dessemontet, R., Bless, G., \& Morin, D. (2012). Effects of inclusion on the academic achievement and adaptive behaviour of children with intellectual disabilities. Journal of intellectual disability research: JIDR, 56(6), 579-587.

Stevens, R. J., \& Slavin, R.E. (1995). The cooperative elementary school: effects on students' achievement, attitudes, and social relations. American Educational Journal, 32(2), 321-325.

Stöckli, M. (2019). Unterrichtsintegrierte Förderung im Mathematikunterricht: Eine empirische Studie in der Primarschule. Dissertation, Universität Zürich. https://www.zora.uzh.ch/id/eprint/177335/. Zugegriffen: 05.05.2020.

Stöckli, M., Moser Opitz, E., Pfister, M., \& Reusser, L. (2014). Gezielt fördern, differenzieren und trotzdem gemeinsam lernen. Überlegungen zum inklusiven Mathematikunterricht. Sonderpädagogische Förderung heute, 59(1), 44-56. 
Waldron, N.L., \& McLeskey, J. (1998). The effect of an inclusive school program on students with mild and severe learning disabilities. Exceptional Children, 64(3), 395-405.

Werner, B. (2019). Mathematik inklusive. Grundriss einer inklusiven Fachdidaktik. Stuttgart: Kohlhammer.

Werner, B., \& Berg, M. (2014). Sprache im Mathematikunterricht - Stolpersteine oder Ressource? https:// core.ac.uk/download/pdf/83041501.pdf. Zugegriffen: 24.05.2020.

Willrodt, K., \& Claybrook, S. (1995). Effects of inclusion on academic outcomes. ERIC Document Reproduction Service No. ED 389 102. Houston: Sam Houston State University.

Winter, H. (2001). Fundamentale Ideen in der Grundschule. https://grundschule.bildung-rp.de/fileadmin/ user_upload/grundschule.bildung-rp.de/Downloads/Mathemathik/Winter_Inhalte_math_Lernens. pdf. Zugegriffen: 18.05.2020.

Wittmann, E.C. (1990). Wider die Flut der „,bunten Hunde“ und der „grauen Päckchen“: Die Konzeption des aktiv entdeckenden Lernens und des produktiven Übens. In E.C. Wittmann \& G. N. Müller (Hrsg.), Handbuch produktiver Rechenübungen. Vom Einspluseins zum Einmaleins (Bd. 1, S. 152-166). Stuttgart: Klett.

Wittmann, E.C. (1998). Standard number representations in the teaching of arithmetic. Journal für Mathematik-Didaktik, 19(2/3), 149-178.

Wittmann, E. C., \& Müller, G. N. (2004). Das Zahlenbuch 1. Lehrerband. Stuttgart: Klett.

Wittmann, E. C., Müller, G. N., Nührenbörger, M., \& Schwarzkopf, R. (Hrsg.). (2017a). Das Zahlenbuch 1. Schülerbuch. Stuttgart: Klett.

Wittmann, E. C., Müller, G. N., Nührenbörger, M., \& Schwarzkopf, R. (Hrsg.). (2017b). Das Zahlenbuch 2. Schülerbuch. Stuttgart: Klett.

Wittmann, E.C., Müller, G. N., Nührenbörger, M., \& Schwarzkopf, R. (Hrsg.). (2018). Das Zahlenbuch 3. Schülerbuch. Stuttgart: Klett.

Wittmann, E. C., Müller, G. N., Nührenbörger, M., \& Schwarzkopf, R. (Hrsg.). (2019). Das Zahlenbuch 4. Schülerbuch. Stuttgart: Klett.

Wocken, H. (1987). Schulleistungen in Integrationsklassen. In H. Wocken \& G. Antor (Hrsg.), Integrationsklassen in Hamburg (S. 276-306). Oberbiel: Jarick. 